\title{
FLORÍSTICA DO ESTRATO ARBUSTIVO-ARBÓREO DE UMA ÁREA DE CERRADO SENSU STRICTO, GURUPI, TOCANTINS
}

Jacqueline Bonfim e Cândido ${ }^{1,2}$, Dayane Pereira Lima1, Paulo Ricardo Teixeira ${ }^{1,2}$, Priscila Bezerra de Souza ${ }^{1,3,4}$

1 -Universidade Federal do Tocantins, Campus de Gurupi. Rua Badejós, Chácaras 69 e 72 - CEP: 77402-970 - Caixa Postal 66 | 77402-970 | Gurupi/TO, Brasil.

2 - Mestrandos em Ciências Florestais e Ambientais da UFT, Campus de Gurupi TO

3 - Docente do Curso de Engenharia Florestal da Universidade Federal do Tocantins, Campus de Gurupi - TO. Bióloga, doutora em Botânica. 4 - Autor para correspondência: priscilauft@uft.edu.br

Recebido em: 03/10/2016 - Aprovado em: 21/11/2016 - Publicado em: 05/12/2016 DOI: 10.18677/EnciBio_2016B_160

\section{RESUMO}

O Cerrado é o segundo maior bioma brasileiro em extensão, com cerca de 204 milhões de hectares, ocupando aproximadamente $22 \%$ do território nacional. 0 trabalho foi desenvolvido em uma área de Cerrado sensu stricto inserido na Reserva Legal da Fazenda Experimental da UFT, campus de Gurupi - TO e teve como objetivo realizar um estudo da florística e fitossociologia do estrato arbustivo-arbóreo de uma área de cerrado sensu stricto, Gurupi, Tocantins. Foram instaladas cinco parcelas de $20 \times 50 \mathrm{~m}$. Todos os indivíduos arbóreos, com diâmetro $\geq$ a $10 \mathrm{~cm}$ medidos $1,30 \mathrm{~cm}$ do solo, foram amostrados. Foram amostrados um total de 906 indivíduos, distribuídos em 97 espécies, incluídas em 76 gêneros, pertencentes a 39 famílias. Os índices de diversidade foram expressivos $\left(H^{\prime}=3,69\right)$ e $\left(J^{\prime}=0,81\right)$, denotando uma elevada biodiversidade local, além de refletir um bom estado de conservação desse fragmento de cerrado sensu stricto.

PALAVRAS-CHAVE: diversidade, flora lenhosa, região norte

\section{FLORISTIC OF ESTRATO THE WOODY OF A CERRADO AREA SENSU STRICTO, GURUPI, TOCANTINS}

\begin{abstract}
The Cerrado is the second largest Brazilian biome, with about 204 million hectares, occupying approximately $22 \%$ of the national territory. The study was conducted in a strict sense Cerrado inserted in the Legal Reserve of Experimental UFT Farm campus Gurupi - TO and aimed to carry out a study of the floristic and phytosociology of shrub-tree layer of a cerrado area, Gurupi, Tocantins. They were installed five plots of $20 \times 50 \mathrm{~m}$. All trees with a diameter $\geq 10 \mathrm{~cm}$ measured $1.30 \mathrm{~cm}$ of soil were sampled. We sampled 906 individuals, distributed in 97 species, included in 76 genera belonging to 39 families. The diversity indices were significant $\left(H^{\prime}=\right.$
\end{abstract}


3.69) and $\left(\mathrm{J}^{\prime}=0.81\right)$, indicating a high local biodiversity, and reflect a good state of conservation of this cerrado fragment.

KEYWORDS: woody flora, floristic survey, north region

\section{INTRODUÇÃO}

O Cerrado é o segundo maior bioma da América do Sul, ocupando uma área de $2.036 .448 \mathrm{~km}^{2}$, cerca de $22 \%$ do território nacional (BRASIL, 2016). A maior parte está localizada no Planalto Central Brasileiro que, conforme a denominação, compreende regiões de elevadas altitudes, na porção central do país (SANTOS, 2015). A vegetação de Cerrado brasileiro compreende um gradiente natural de fisionomias, de campos abertos a savanas e florestas, dependendo das condições do solo e da disponibilidade de água no local (SILVA et al., 2015).

A flora dos remanescentes de Cerrado encontra-se bastante ameaçada, pois espécies nativas endêmicas estão desaparecendo ao longo do tempo, seja pelas pressões antrópicas (OLIVEIRA et al., 2015), seja pela supressão total de distúrbios como o fogo (DURIGAN \& RATTER, 2006). Como ocorre em outros Estados, o Tocantins sofre também processos de alterações ambientais, principalmente em decorrência das atividades antrópicas realizadas de maneira insustentável. Originalmente, $91 \%$ do território tocantinense era coberto por Cerrado (SANO et al., 2010), atualmente apenas $72 \%$ dessa cobertura original encontra-se preservada, todavia é considerado o Estado que apresenta os maiores índices de vegetação remanescente do domínio Cerrado (BRASIL, 2015).

Nesse contexto, trabalhos florísticos representam possibilidades de conhecer qualitativa e quantitativamente a flora local, fornecendo base para pesquisas posteriores, que podem ser usadas para o desenvolvimento de estratégias e/ou iniciativas de conservação e recuperação de áreas que tenham sido alteradas (ABREU et al., 2014).

Diante da importância dos levantamentos florísticos para o reconhecimento da diversidade biológica e distribuição das espécies no domínio do Cerrado, objetivouse realizar um estudo da florística e fitossociologia do estrato arbustivo-arbóreo de uma área de cerrado sensu stricto, Gurupi, Tocantins.

\section{MATERIAL E MÉTODOS}

O trabalho foi desenvolvido em área de cerrado sensu stricto inserido na Reserva Legal da Fazenda Experimental da UFT, campus de Gurupi - TO, sob as coordenadas UTM 1146'25" S e 4902'54" W de apro ximadamente 138 hectares.O clima da região é caracterizado como tropical úmido com estação chuvosa no verão e seca no inverno. A temperatura média ao longo do ano varia entre $22^{\circ} \mathrm{C}$ e $28^{\circ} \mathrm{C}$, já a precipitação varia entre $1.500 \mathrm{~mm}$ a 1.600mm (SEPLAN, 2012). A altitude registrada nas áreas avaliadas está situada entre as cotas de 250 a 300 metros. $O$ solo é classificado como Plintossolo (EMBRAPA, 2013).

O estudo foi realizado no período de setembro de 2014 a agosto de 2015, utilizando-se o método de parcela (MUELLER-DOMBOIS \& ELLENBERG, 1974). Foram alocadas, sistematicamente, cinco parcelas permanentes, de $20 \times 50 \mathrm{~m}$, ou seja, $1000 \mathrm{~m}^{2}$ cada, sendo que as mesmas foram distanciadas $20 \mathrm{~m}$ entre si, totalizando uma área amostral de 0,5 ha. No interior das parcelas, foram amostrados todos os indivíduos arbustivo-arbóreos, com circunferência a 1,30m do solo (CAP) $\geq$ a $10 \mathrm{~cm}$. 
A identificação taxonômica das espécies foi realizada em campo, caso contrário o material botânico era coletado para posterior identificação por meio de comparações com o material do Herbário da UFT, campus de Porto Nacional, literatura especializada e consultas a especialistas. $O$ sistema de classificação adotado foi o "Angiosperm Phylogeny Group" (APG III, 2009). Todos os nomes das espécies e os respectivos autores e sinonímias foram confirmados e atualizados pelo site da Lista de Espécies da Flora do Brasil (FLORA DO BRASIL 2020, 2016).

Para obtenção dos parâmetros fitossociológicos: densidade relativa (DR), densidade absoluta (DA), dominância relativa (DoR), dominância absoluta (DoA), frequência absoluta ( $F A)$, frequência relativa $(F R)$ e índice de valor de importância (IVI). Para obter o índice de Shannon-Weaver $\left(\mathrm{H}^{\prime}\right)$ que representa o índice de diversidade; a equabilidade de Pielou para a uniformidade (J') BROWER \& ZAR (1984) utilizou-se o software Fitopac versão 2.1.2 (SHEPHERD, 2010).

\section{RESULTADOS E DISCUSSÃO}

Foram amostrados em 0,5 ha de uma área de cerrado sensu stricto um total de 906 indivíduos, distribuídos em 97 espécies, incluídas em 76 gêneros, pertencentes a 39 famílias. O total de espécies amostradas na área está dentro do intervalo de 25 a 102 espécies normalmente encontradas no Cerrado do Tocantins (SANTOS \& LÓLIS, 2007; FERREIRA et al., 2015; SILVA NETO et al., 2016). Dessa forma pode-se inferir que o número de espécies encontradas está de acordo com os padrões de riqueza para o estrato lenhoso das áreas de cerrado sensu stricto, ou seja, não ultrapassam 120 espécies (RATTER et al., 1997).

Os valores de riqueza observados para a área avaliada corroboram com o encontrado em outra área de cerrado sensu stricto do Município de Gurupi (FERREIRA et al., 2015). Além disso, observou-se um valor próximo a área de cerradão no Município de Pium (SANTOS \& LÓLIS, 2007) (Tabela 1). Pode-se concluir que a área de cerrado sensu stricto estudada, possui uma florística diversificada, semelhante aos cerradões (ALVES et al., 2013).

TABELA 1: Comparação dos valores de riqueza com outros estudos realizados em áreas de cerrado sensu stricto e em outras fisionomias em diferentes localidades do Brasil, em que: $A$ = área amostral em hectares, $C . I=$ critério de inclusão adotado, $\mathrm{N}^{\circ} \mathrm{sp}=$ número de espécies, $\mathrm{N}^{\circ}$ gên = númer $\mathrm{o}$ de gêneros, $\mathrm{N}^{\circ}$ fam $=$ número de famílias

\begin{tabular}{|c|c|c|c|c|c|c|}
\hline Áreas de estudo & $\dot{A}$ (ha) & C. I & Fisionomia & $N^{\circ} s p$ & $N^{\circ}$ gên & $\mathbf{N}^{\circ}$ fam \\
\hline Gurupi - TO ** & 0.5 & $\mathrm{CAP} \geq 10$ & Cerrado s.s & 97 & 76 & 39 \\
\hline Gurupi - TO ${ }^{1}$ & 0,5 & $\mathrm{CAP} \geq 10$ & Cerrado s.s & 102 & 78 & 42 \\
\hline Dueré - TO ${ }^{2}$ & 0,2 & $\mathrm{CAP} \geq 15$ & Cerrado s.s & 41 & - & 21 \\
\hline Caseara $-\mathrm{TO}^{3}$ & 0,2 & $\mathrm{CAP} \geq 10$ & Cerrado s. s & 25 & - & 17 \\
\hline Pium $-\mathrm{TO}^{3}$ & 0,5 & $\mathrm{CAP} \geq 10$ & Cerradão & 77 & - & 37 \\
\hline $\begin{array}{l}\text { Chapada dos } \\
\text { Guimarães e Baixada } \\
\text { Cuiabana - MT }{ }^{4}\end{array}$ & 3,3 & DAS $30 \geq 5$ & Cerrado s. s & 106 & 81 & 36 \\
\hline Jaraguá - $\mathrm{GO}^{5}$ & 1,0 & DAS $30 \geq 5$ & Cerrado s. S & 63 & 51 & 28 \\
\hline
\end{tabular}

** Área do presente trabalho; FERREIRA et al., (2015) ${ }^{1}$; SILVA NETO et al., (2016) ${ }^{2}$; SANTOS \& LÓLIS, (2007) ${ }^{3}$; FINGER \& FINGER (2015) ${ }^{4}$; ABREU et al., (2014).

Entretanto, ao comparar a riqueza com áreas do centro-oeste, o total de espécies encontradas para a área de estudo foi superior ao encontrado por ABREU et al., (2014) em área de cerrado sensu stricto no Estado de Goiás e semelhante ao ENCICLOPÉDIA BIOSFERA, Centro Científico Conhecer - Goiânia, v.13 n.24; p.1746 2016 
amostrado por FINGER \& FINGER (2015) em área cinco vezes maior cerca de 3 ha de cerrado sensu stricto no Estado do Mato Grosso (Tabela 1).

Dessa forma, foi possível observar que o presente estudo apresentou uma riqueza superior ao descrito por FELFILI \& SILVA JÚNIOR (1993) para os limites de riqueza de espécies lenhosas em áreas de cerrado sensu stricto na região do Planalto Central brasileiro, entre 50 e 80 espécies. Segundo HAIDAR et al., (2008), variações das características ambientais, como solo, relevo e proximidade com bacias hidrográficas, geram grande influência na composição florística e estrutura das comunidades de cerrado sensu stricto, podendo explicar a diferença no padrão de riqueza dessas regiões.

Diante disso, embora a área de estudo possua uma considerável riqueza de espécies quando comparadas a outras áreas (Tabela 1) existem dois fatores que podem ter inferido nesta constatação, ou seja, o tamanho das áreas avaliadas nos diferentes estudos podem determinar ou não a ocorrência de um número maior ou menor de espécies e as diferentes metodologias adotadas como: formas de parcelas e critério de inclusão dificultando a interpretação de eventuais padrões que venham a ser detectados na comunidade (GASPAR et al., 2014).

As famílias com maior riqueza em número de espécies foram Fabaceae (12), Myrtaceae (7), Annonaceae (6), Apocynaceae, Chrysobalanaceae e Rubiaceae, (5), Anacardiaceae, Malpighiaceae, Malvaceae e Vochysiaceae (4), Bignoniaceae, Meliaceae e Moraceae (3), Connaraceae, Dilleniaceae, Ebenaceae, Ochnaceae, Primulaceae e Sapindaceae (2) as demais famílias contribuíram com apenas uma espécie cada, dessa forma das 39 espécies presentes na área de estudo $51 \%$ foram representadas por uma única espécie.

Com relação à flora do cerrado sensu stricto, as famílias como Fabaceae e Myrtaceae, geralmente apresentam altos valores de riqueza florística corroborando com o encontrado em outros trabalhos (ABREU et al., 2014; ALMEIDA et al., 2014; FINGER \& FINGER, 2015; SILVA NETO et al., 2016).

A família Fabaceae não se destaca somente no Cerrado, mas em outras formações vegetais, tanto em riqueza específica quanto em valores de importância. Dessa forma, é considerada uma das famílias mais representativas na flora do Cerrado, já que está presente com altas densidades em todas as fitofisionomias desse bioma (HERINGER et al., 1977). O sucesso adaptativo das espécies da família Fabaceae pode estar relacionado ao estabelecimento de relações simbióticas com microrganismos, que potenciam a capacidade de absorção dos elementos fósforo e nitrogênio, que são encontrados em baixas concentrações nos solos oligotróficos como os do Cerrado, tais elementos são fundamentais para 0 crescimento e estabelecimento das espécies (AQUINO et al., 2014).

As espécies com maior representatividade quanto ao número de indivíduos foram Myrcia splendens (Sw.) DC. (137), Qualea multiflora Mart. (68), Protium heptaphyllum (Aubl.) Marchand (63), Magonia pubescens A.St- Hil. (42), Qualea grandiflora Mart. (31), Tapirira guianensis Aubl. (28), Qualea parviflora Mart. (28), Byrsonima pachyphylla A. Juss. (28) e Coccoloba mollis Casar. (25), as demais espécies apresentaram valores abaixo de 20 indivíduos cada, resultados estes que corroboram com FERREIRA et al. (2015). Segundo RATTER et al. (1997), o gênero Qualea é considerado abundante em áreas de Cerrado, gerando assim ampla dominância e distribuição desse gênero.

As famílias que apresentaram os maiores índices de valores de importância (IVI) foram Myrtaceae (10,06\%); Vochysiaceae (8,99\%); Burseraceae (7,48\%), 
Fabaceae (6,87\%) e Anacardiaceae (5,33\%), somando juntas 38,73\% do IVI total, esses resultados corroboram com outros trabalhos feitos em áreas de cerrado sensu stricto (FINGER \& FINGER, 2015; OLIVEIRA et al., 2015; SILVA NETO et al., 2016).

A família Burseraceae apresentou altos valores de IVI, devido a espécie Protium heptaphyllum única espécie da família amostrada, essa espécie apresentou o terceiro maior valor em número de indivíduos (63), segundo maior valor de IVI $(6,97 \%)$ e maiores valores de DoR e DoA, corroborando com SANTOS \& LÓLIS, 2007, realizado no Estado do Tocantins, quando encontraram altos valores de IVI para a família Burseraceae em diferentes áreas de cerradão.

Já as espécies que tiveram maior IVI foram Myrcia splendes (7,87\%), Protium heptaphyllum (6,97\%), Qualea multiflora (4,65\%), Magonia pubescens $(4,09 \%)$ e Tapirira guianensis (3,37\%), somando juntas $26,95 \%$ do IVI total. FERREIRA et al. (2015), encontraram quatro dessas cinco espécies com os maiores valores de IVI. SILVA NETO et al. (2016), em Dueré - TO e ABREU et al. (2014), em Jaraguá - GO, apresentaram espécies do gênero Qualea com os maiores valores de IVI, corroborando com o presente estudo.

Do total de 97 espécies, cinco apresentaram IVI maior que 3\%, em contrapartida, 67 espécies tiveram valores inferiores a 1\%. Um grande número de espécies com índices de valor de importância baixo é uma característica das florestas tropicais, ou seja, poucas espécies detêm altos valores relativos de densidade, frequência e dominância (SILVA NETO et al., 2016).

Entre todas as parcelas amostradas em 0,5 ha de cerrado sensu stricto, 13 espécies apresentaram $100 \%$ de frequência absoluta, sendo estas Myrcia splendens (Sw.) DC., Protium heptaphyllum (Aubl.) Marchand, Qualea multiflora Mart., Magonia pubescens A.St- Hil., Tapirira guianensis Aubl., Qualea parviflora Mart., Luehea grandiflora Mart. \& Zucc., Coccoloba mollis Casar., Copaifera langsdorffii Desf., Vatairea macrocarpa (Benth.) Ducke, Xylopia aromatica (Lam.) Mart., Astronium fraxinifolium Schott, Roupala montana Aubl.

O Índice de Diversidade de Shannon-Wiener $\left(\mathrm{H}^{\prime}\right)$ calculado na área foi de 3,69 e a equabilidade de Pielou (J') de 0,81, demostrando assim uma alta riqueza e diversidade, apresentando forte heterogeneidade ambiental e baixa dominância ecológica, valores considerados altos, refletindo assim um bom estado de conservação (Tabela 2).

TABELA 2: Comparação dos valores de diversidade com outros estudos realizados em áreas de cerrado sensu stricto e em outras fisionomias em diferentes localidades do Brasil, em que: $A$ = área amostral em hectares, $C . I=$ critério de inclusão adotado, $\mathrm{H}^{\prime}$ = índice de diversidade de Shannon-Weaver, J' = índice de equabilidade de Pielou

\begin{tabular}{|c|c|c|c|c|c|}
\hline Áreas de estudo & Á (ha) & C. I & Fisionomia & $\left(H^{\prime}\right)$ & $\left(J^{\prime}\right)$ \\
\hline Gurupi - TO ** & 0,5 & $\mathrm{CAP} \geq 10$ & Cerrado s.s & 3,69 & 0,81 \\
\hline Gurupi - TO ${ }^{1}$ & 0,5 & $\mathrm{CAP} \geq 10$ & Cerrado s.s & 3,70 & 0,80 \\
\hline Dueré - TO ${ }^{2}$ & 0,2 & $\mathrm{CAP} \geq 15$ & Cerrado s.s & 3,21 & 0,86 \\
\hline Caseara - $\mathrm{TO}^{3}$ & 0,2 & $\mathrm{CAP} \geq 10$ & Cerrado s. s & 3,54 & 0,85 \\
\hline Pium - $\mathrm{TO}^{3}$ & 0,5 & $\mathrm{CAP} \geq 10$ & Cerradão & 3,67 & 0,84 \\
\hline $\begin{array}{l}\text { Chapada dos } \\
\text { Guimarães e Baixada } \\
\text { Cuiabana - MT }{ }^{4}\end{array}$ & 3,3 & DAS $30 \geq 5$ & Cerrado s. s & 4,03 & - \\
\hline Jaraguá - GO ${ }^{5}$ & 1,0 & DAS $30 \geq 5$ & Cerrado s. s & 3,41 & 0,82 \\
\hline
\end{tabular}

** Área do presente trabalho; FERREIRA et al., (2015) ${ }^{1}$; SILVA NETO et al., (2016) ${ }^{2}$; SANTOS \&

LÓLIS, (2007) 3; FINGER \& FINGER (2015) ${ }^{4}$; ABREU et al., (2014). 
Segundo MARTINS (1991), os valores de diversidade de Shannon variam de 1,5 a 3,5 nats/ind., raramente passando de 4,5. Dessa forma, pode-se inferir que os índices encontrados no presente trabalho estão muito próximos aos definidos por diferentes autores em trabalhos realizados em diferentes regiões do Tocantins (Tabela 2). Ao avaliarem uma área de cerrado sensu stricto no Munícipio de Gurupi - TO, FERREIRA et al. (2015), encontraram valores semelhantes para a diversidade de Shannon $\left(H^{\prime}=3,70\right)$ e para equabilidade de Pielou $\left(J^{\prime}=0,80\right)$, utilizando o mesmo esforço amostral e critério de inclusão deste trabalho. Valores próximos foram encontrados no Município de Dueré, Caseara e Pium (SANTOS \& LÓLIS, 2007; SILVA NETO et al., 2016).

No Estado de Goiás os valores encontrados foram semelhantes ao do presente estudo apesar de um critério de inclusão diferente, em contrapartida em um cerrado sensu stricto do Mato Grosso, encontrou-se valores para a diversidade de Shannon considerados superiores aos comumente encontrados para esta tipologia vegetal $\left(H^{\prime}=4,03\right)$ (FINGER \& FINGER, 2015).

Todavia, deve-se levar em consideração que alguns trabalhos podem adotar diferentes metodologias como: formas de parcelas, critério de inclusão, dificultando a interpretação de eventuais padrões que venham a ser detectados na comunidade (GASPAR et al., 2014), consequentemente a interpretação dos resultados e comparação com outros trabalhos.

Dessa forma, o valor do índice de diversidade de Shannon do presente estudo, está dentro do intervalo apresentado para outras áreas de Cerrado do Tocantins, Goiás e Mato Grosso 3,21 a 4,03 e índice de equabilidade de Pielou 0,80 a 0,86 (Tabela 2). Esse alto valor demostra elevada biodiversidade local e reflete a estrutura da comunidade em termos da abundância relativa, das diferentes espécies que compõem a riqueza (FINGER \& FINGER, 2015).

\section{CONCLUSÕES}

Os valores de riqueza e índices de diversidade foram expressivos, denotando uma elevada biodiversidade local, dessa forma pode-se inferir que o fragmento de cerrado sensu stricto evidencia um bom estado de conservação.

Entretanto, torna-se necessário novas pesquisas relacionadas a riqueza e diversidade florística no Estado do Tocantins, para auxiliar futuramente no desenvolvimento de estratégias e/ou iniciativas de conservação e recuperação de áreas que tenham sido alteradas no domínio do Cerrado Tocantinense.

\section{REFERÊNCIAS}

ABREU, T.A.L.; PINTO, J.R.R.; LENZA, E.; MEWS, H.A.; DOS SANTOS, T.R.R. Composição florística e estrutura da vegetação arbustivo-arbórea em Cerrado sentido restrito na Serra de Jaraguá, Goiás, Brasil. Heringeriana, v. 6, n. 2, p. 42-53, $2014 . \quad$ Disponível em: <http://portalinseer.ibict.br/index.php/heringeriana/article/view/29> Acesso em: 30 set. 2016.

ALMEIDA, R.F.; FAGG, C.W.; OLIVEIRA, M.C.; MUNHOZ, C.B.R.; LIMA, A.S.; OLIVEIRA, L.S.B. Mudanças florística e estruturais no cerrado sensu stricto ao longo de 27 anos (1985-2012) na Fazenda Água Limpa, Brasília, DF. Revista Rodriguésia, v.65, n.1, p.01-19, 2014. Disponível em: < http://rodriguesiaseer.jbrj.gov.br/index.php/rodriguesia/article/view/759> Acesso em: 30 set. 2016. 
ALVES H.R.; PRADO JÚNIOR, J.A.; LOPES, S.F.; SILVA, P.P.F.; PEPPE, F.B.; SCHIAVINI, I. Fitossociologia e grupos ecológicos da comunidade lenhosa em um remanescente de cerradão em Uberlândia, MG. Revista Caminhos de Geografia, v.14, n.46, p. 236-245, 2013. Disponível em: <http://www.seer.ufu.br/index.php/caminhosdegeografia/article/view/22473> Acesso em: 30 set. 2016.

ANGIOSPERM PHYLOGENY GROUP - APG. An update of the Angiosperm Phylogeny Group classification for the orders and families of flowering plants: APG III. Bot. J. Linn. Soc., v.161, p.105-121. 2009. Disponível em: <http://onlinelibrary.wiley.com/doi/10.1111/j.1095-8339.2009.00996.x/epdf>. Acesso em: 09 ago. 2015.

AQUINO, F.G.; PEREIRA, C.S.; PASSOS, F.B.; DE OLIVEIRA, M.C. Composição florística e estrutural de um cerrado sentido restrito na área de proteção de Manancial Mestre D'Armas, Distrito Federal. Bioscience Journal, v. 30, n. 2, 2014. Disponível em: <http://www.seer.ufu.br/index.php/biosciencejournal/article/view/18305/13775> Acesso em: 30 set. 2016.

BRASIL. MMA - Ministério do Meio Ambiente. Bioma Cerrado - 2016. Disponível em: <http://www.mma.gov.br/biomas/cerrado>. Acesso em: 31 ago. 2016.

BRASIL. MMA - Ministério do Meio Ambiente. Mapeamento do Uso e Cobertura do Cerrado: Projeto TerraClass Cerrado 2013. Brasília: MMA, 2015, p.42-47. ISBN 97885-7738-255-2

BROWER, J.E.; ZAR, J.H. Field \& laboratory methods for general ecology. 2 ed. Wm. C. Brown Publishers, Dubuque, lowa, 1984. p.226.

DURIGAN, G.; RATTER, J. A. Successional changes in Cerrado and Cerrado/Forest ecotonal vegetation in western Sao Paulo State, Brazil, 1962-2000. Edinburgh Journal of Botany, 63: 119-130, 2006. Disponível em: < https://www.cambridge.org/core/journals/edinburgh-journal-of-

botany/article/successional-changes-in-cerrado-and-cerradoforest-ecotonal-

vegetation-in-western-sao-paulo-state-brazil-

19622000/9676B71E61DE8D94E4E80A557CA838E8> Acesso em: 30 set. 2016. DOI: http://dx.doi.org/10.1017/S0960428606000357

EMBRAPA - EMPRESA BRASILEIRA DE PESQUISA AGROPECUÁRIA. Sistema brasileiro de classificação de solos. Centro Nacional de Pesquisas de Solos. 3.ed. Rio de Janeiro, 2013. 353p.

FELFILI, J.M.; SILVA-JUNIOR, M.C. A comparative study of cerrado (sensu stricto) vegetation in Central Brazil. Journal Tropical of Ecology, v.9, n.3, p.277-289, 1993. Disponível em: <https://www.cambridge.org/core/journals/journal-of-tropicalecology/article/a-comparative-study-of-cerrado-sensu-stricto-vegetation-in-central- 
brazil/4BDB89875EDFEE7E6C4AE7E59B3D2B4A> Acesso em: 30 set. 2016. DOI: http://dx.doi.org/10.1017/S0266467400007306

FINGER, Z.; FINGER, F. A. Fitossociologia em comunidades arbóreas remanescentes de cerrado sensu stricto no brasil central. Floresta, v. 45, n. 4, p. 769-780, 2015. Disponível em: <http://revistas.ufpr.br/floresta/article/view/30860> Acesso em: 30 set. 2016. DOI: http://dx.doi.org/10.5380/rf.v45i4.30860

FERREIRA, Q. S. F.; OLIVEIRA, C. M.; SOUZA, P. B.; ANDRADE, V. C. L.

Fitossociologia e estrutura diamétrica de um cerrado sensu stricto, Gurupi - TO. Revista Verde de Agroecologia e Desenvolvimento sustentável, v.10, n.1, p. 229-235, $2015 . \quad$ Disponível em: <http://www.gvaa.org.br/revista/index.php/RVADS/article/view/2996> Acesso em: 30 set. 2016. DOI: http://dx.doi.org/10.18378/rvads.v10i1.2996

FLORA DO BRASIL 2020. Jardim Botânico do Rio de Janeiro. Disponível em: <http://floradobrasil.jbrj.gov.br/ >. Acesso em: 08 Set. 2016.

GASPAR, R.D.O.; CASTRO, R.V.O.; PELOSO, R.V.D.; SOUZA, F.C.D., MARTINS, S.V. Phytosociological and carbon stock analysis in the tree layer of a semideciduous forest fragment. Ciência Florestal, v. 24, n. 2, p. 313-324, 2014. Disponível em: $<$ http://www.scielo.br/scielo.php?pid=S1980-

50982014000200313\&script=sci_arttext\&tlng=pt> Acesso em: 30 set. 2016.

DOI: http://dx.doi.org/10.5902/1980509814569

HAIDAR, R.F.; AMARAL, A.G.; BRANDÃO, M.M.; CARNEIRO, D.C.; MARTINS, R.C.; DE QUEIROZ MATOS, M.; PIERUCCETTI, R.L.G.; LAGO, F.P.D.L.S. Fitossociologia e diversidade do cerrado sobre solo raso na Estação Ecológica do Jardim Botânico de Brasília e sua relação com outros cerrados em áreas protegidas do Distrito Federal. Heringeriana, v. 2, n. 2, p. 43-60, 2008. Disponível em: <http://portalinseer.ibict.br/index.php/heringeriana/article/view/101> Acesso em: 16 set. 2016.

HERINGER, E.P.; BARROSO, G.M.; RIZZO, J.A.; RIZZINI, C.T. A flora do Cerrado. In: SIMPÓSIO SOBRE CERRADO, 4. São Paulo: Editora da Universidade de São Paulo,15-36p, 1977.

MARTINS, F.R. Estrutura de uma floresta mesófila. São Paulo: Ed da UNICAMP, 1991. $246 \mathrm{p}$.

MUELLER-DOMBOIS, D.Y.; ELLENBERG, M. Aims and methods in vegetation ecology. New York: John Wiley \& Sons, 1974. 547p.

OLIVEIRA, C.P.; FRANCELINO, M.R.; CYSNEIROS, V.C.; DE ANDRADE, F.C.; BOOTH, M.C. Composição florística e estrutura de um cerrado sensu stricto no oeste da Bahia. CERNE, v. 21, n. 4, p. 545-552, 2015. Disponível em: <http://www.redalyc.org/articulo.oa?id=74444232004> Acesso em: 30 set. 2016. DOI: $10.1590 / 01047760201521041722$ 
RATTER, J.A.; RIBEIRO, J.F.; BRIDGEWATER, S. The Brazilian cerrado vegetation and threats to its biodiversity. Annals of Botany, n.80, p.223-230, 1997. Disponível em: < http://aob.oxfordjournals.org/content/80/3/223.short> Acesso em: 30 set. 2016.

SANO, E.E.; ROSA, R.; BRITO, J.L.S.; FERREIRA, L.G. Land cover mapping of the tropical savanna region in Brazil. Environmental Monitoring and Assessment, $v$. 166, 113-114, 2010. Disponível em: <http://link.springer.com/article/10.1007/s10661009-0988-4> Acesso em: 20 set. 2016. DOI: 10.1007/s10661-009-0988-4

SANTOS, C. O espírito do capitalismo na ocupação dos cerrados brasileiros nos estados da Bahia e do Piauí. GOT, Revista de Geografia e Ordenamento do Território, n. $8, \quad$ p. 229-253, 2015. Disponível em: <http://www.scielo.mec.pt/scielo.php?script=sci_arttext\&pid=S218212672015000200013> Acesso em: 20 set. 2016. DOI: http://dx.doi.org/10.17127/got/2015.8.012

SANTOS, E.R.; LÓLIS, S.F. Análise florística em comunidades florestais nos Municípios de Caseara, Marianópolis, e Pium, no estado do Tocantins. Revista Carbono Social, v.01, n.02, p. 24-31, 2007. Disponível em: < http://docplayer.com.br/13596691-Revista-carbono-social.html> Acesso em: 20 set. 2016.

SEPLAN. Atlas do Tocantins: subsídios ao planejamento da gestão territorial, Palmas. Atual, v.6, 2012.

SILVA, R.B.M.D.; FRANCELINO, M.R.; MOURA, P.A.; MOURA, T.A.; PEREIRA, M.G.; OLIVEIRA, C.P.D. Soil-vegetation relation in cerrado enviroment under influence of the group Urucuia. Ciência Florestal, v. 25, n. 2, p. 363-373, 2015. Disponível em: <http://www.scielo.br/scielo.php?pid=S198050982015000200363\&script=sci_arttext> Acesso em: 20 set. 2016. DOI: http://dx.doi.org/10.5902/1980509818455

SHEPHERD, G.J. FITOPAC 2: manual do usuário. Campinas: UNICAMP, 2010. $91 \mathrm{p}$.

SILVA NETO, V.L.; OLIVEIRA, A.L.; FERREIRA, R.Q.S.; SOUZA, P.B.; VIOLA, M.R. Fitossociologia e distribuição diamétrica de uma área de cerrado sensu stricto, Dueré - TO. Revista de Ciências Ambientais, v. 10, n. 1, p. p. 91-106, 2016. Disponível em: <http://www.revistas.unilasalle.edu.br/index.php/Rbca/article/view/2471> Acesso em: 20 set. 2016. 